\title{
REPRESENTACIONES RUPESTRES DEL NORESTE DE NEUQUÉN (PATAGONIA SEPTENTRIONAL). PRIMERAS TENDENCIAS ESPACIALES Y TEMPORALES
}

\author{
ROCK ART FROM NORTHEAST NEUQUÉN PROVINCE (NORTHERN \\ PATAGONIA, ARGENTINA). FIRST SPATIAL AND TEMPORAL TRENDS
}

\author{
Guadalupe Romero' ${ }^{1}$ y Anahí $\operatorname{Re}^{2}$ \\ 1. CONICET-INAPL, 3 de Febrero 1378 (C1426BJN) Ciudad Autónoma de Buenos Aires, \\ E-mail: guada.romero.arq@gmail.com \\ 2. CONICET-UBA-INAPL, 3 de Febrero 1378 (C1426BJN) Ciudad Autónoma de Buenos Aires. \\ E-mail: anahire1@gmail.com
}

Presentado el: 31/10/2013 - Aceptado el: 07/05/2014

\begin{abstract}
Resumen
En este trabajo se realiza una primera caracterización de las representaciones rupestres documentadas en el departamento Pehuenches, noreste de Neuquén (Patagonia septentrional). Partiendo desde un marco de referencia biogeográfico, se discute el potencial rol del arte rupestre estudiado como demarcador simbólico del espacio, evaluándose las características de este registro en espacios con diferentes propiedades ecológicas y geográficas. Luego, se contextualiza esta información en una escala más amplia a partir de una primera comparación con lo registrado por otros investigadores en regiones aledañas. Asimismo, se consideran diversos indicadores cronológicos a fin de realizar una asignación temporal tentativa de las manifestaciones rupestres analizadas. A partir de las tendencias presentadas, se inicia la discusión respecto del rol que ocupó la información y su circulación en la organización de las poblaciones humanas que habitaron el noreste de Neuquén en el pasado.
\end{abstract}

Palabras claves: Arte rupestre, circulación de información, biogeografía, Holoceno tardío.

\footnotetext{
Abstract

This article presents a first analysis of the rock art registered in the Pehuenches department, Neuquén province (northern Patagonia). The possibility of considering these rock art motifs as symbolic
} 
space markers is debated from a biogeographical point of view by evaluating them in relation to spaces of different ecological and geographical properties. This information is preliminary compared in a wider scale doing an analysis of the published data from nearby regions. Also the chronological trend of these representations is reviewed. Finally, the discussion of the role of information and its circulation in the organization of human populations that inhabitated the region in the past, is presented.

Keywords: Rock art, information circulation, biogeography, late Holocene.

\section{Introducción}

En este trabajo se realiza una primera caracterización de las representaciones rupestres documentadas en el departamento Pehuenches, ubicado en el noreste de la provincia de Neuquén (Patagonia septentrional) (Figura 1). Específicamente, en esta oportunidad se realiza un acercamiento inicial a la discusión sobre el rol que ocupó la información y su circulación en la organización de las poblaciones humanas que habitaron la región de estudio. Dicha problemática será abordada a partir del estudio de las manifestaciones rupestres relevadas, en escalas meso y macroregional (sensu Dincauze 2000).

Así, se inicia la discusión respecto del potencial rol del arte rupestre estudiado como demarcador simbólico del espacio, al evaluarse las características de este registro en espacios con diferentes propiedades biogeográficas. Cabe destacar que otros autores desde diferentes marcos teóricos han abordado la circulación de información, la interacción social y la demarcación territorial a partir del estudio de las representaciones rupestres (Barton et al. 1994; Bradley et al. 1994; Belardi et al. 2013; David y Lourandos 1998; Jochim 1983; McDonald y Veth 2011, 2013; Taçon 1994, entre otros). En particular, en este trabajo se adhiere a la propuesta de Belardi y coautores, quienes a partir del concepto de comunicación de Aunger (2009: 37), plantean que las representaciones rupestres constituyen una instancia de construcción de nicho mediante transmisión de información (Re 2010; Scheinsohn 2011).

Cabe destacar que este trabajo se inserta en el marco de un proyecto recientemente iniciado en el departamento Pehuenches que busca generar información sobre el noreste de Neuquén en distintas escalas de análisis, a partir de la integración de diversas líneas de evidencia arqueológica en un marco biogeográfico de referencia (Barberena 2010). A futuro, la perspectiva es articular los resultados alcanzados en el contexto más amplio del poblamiento humano de Patagonia septentrional y regiones aledañas.

A continuación, se caracterizan las representaciones rupestres relevadas tomando en consideración su distribución, densidad y composición. Así, por un lado, se describen los sitios donde son registradas, incluyéndose la ubicación y las dimensiones de los mismos así como las características de los soportes presentes en cada uno de ellos. Por otro lado, se discuten las siguientes variables de los motivos rupestres: categorías y tipos de motivos, técnicas, tonalidades y superposiciones. Dado el estado de las investigaciones, se dispone de información cuantitativa de algunas de ellas mientras que otras, por el momento, sólo serán presentadas en términos cualitativos. 


\section{La región de estudio}

Como se adelantó, la región de estudio corresponde al departamento Pehuenches, ubicado en el extremo noreste de la provincia de Neuquén (Patagonia septentrional) (Figura 1). En escala macro-regional, en la zona comprendida entre los $30^{\circ} \mathrm{S}$ a $40^{\circ} \mathrm{S}$ ocurre la transición de los sistemas de circulación atmosférica Subtropical y Templado, lo que se traduce en un clima árido y semiárido (Páez et al. 2004). A nivel fitogeográfico, se encuentran representadas las Provincias del Monte y la Patagonia (Morrone 2001).

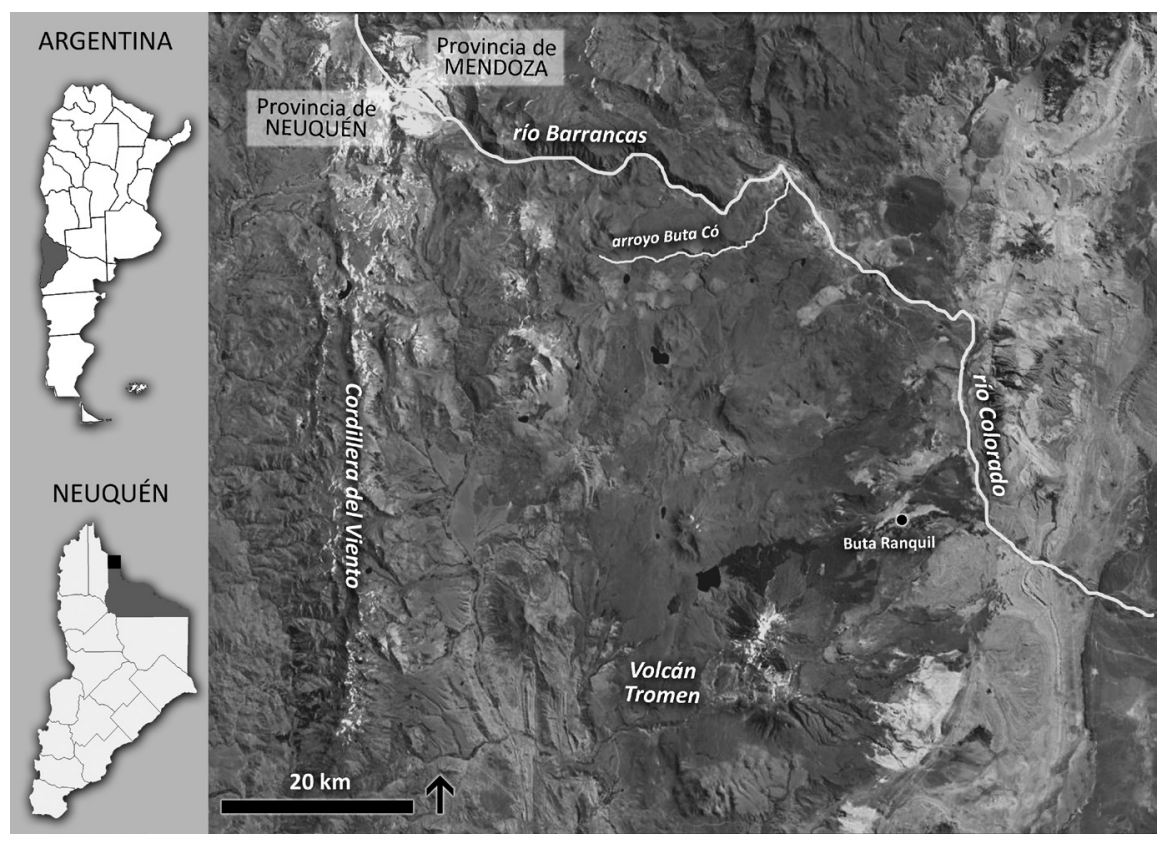

Figura 1. Ubicación de la región de estudio.

La zona bajo estudio se ubica dentro del Campo Volcánico Tromen, caracterizado por una intensa actividad volcánica que ocurrió, fundamentalmente, entre el Plioceno tardío y el Holoceno, incluso durante momentos históricos (Folguera et al. 2008; Galland et al. 2007) (Figura 1). Asimismo, en este ámbito se cuenta con una gran fuente de obsidianas de alta calidad -denominada Huenul- que conforma depósitos secundarios, remanentes de erosión de ignimbritas que las portan (Barberena et al. 2011; Durán et al. 2004). Otro rasgo orográfico destacado que delimita el área bajo estudio hacia al oeste es la cadena montañosa de la Cordillera del Viento, con alturas promedio cercanas a los $3000 \mathrm{msnm}$ y de relieve abrupto cuyos pronunciados desniveles y flancos están surcados por valles angostos y profundos (Fernández 2000 [1979]).

Los ríos más importantes que surcan el área son el Barrancas y el Colorado, que además constituyen los límites norte y este del área bajo estudio (Figura 1). El primero se origina en la Cordillera de los Andes y, en cercanía a la localidad de Buta Ranquil, se une con el río Grande -proveniente de la provincia de Mendoza- para formar el río Colorado. Entre los principales afluentes de este último se cuenta el arroyo Buta Có, en torno al cual se han articulado la mayoría de los trabajos arqueológicos realizados en el área de estudio hasta el momento. 


\section{Antecedentes de investigación}

Respecto del arte rupestre del área de estudio, la información publicada con anterioridad a esta investigación es escasa y sólo se limita a breves referencias (Fernández 1974-1976, 1978; Schobinger 1985). En contraposición, se cuenta con importantes trabajos referidos a esta línea de evidencia en espacios vecinos ubicados a una distancia máxima de $100 \mathrm{~km}$ lineales como: las cuencas de los ríos Neuquén y Curi Leuvú, en el noroeste de Neuquén (Fernández 2000 [1979]; Hajduk y Cúneo 2009; Menghin 1957; Schobinger 1956, entre otros), la Cordillera Andina de Linares, en el centro-este de Chile (Niemeyer y Weisner 1972-1973) y el sur de Mendoza, específicamente en el departamento Malargüe (Gradin 1997-1998; Schobinger 1978, 2002; Tucker y Risi 2010) (Figura 2).

Como se mencionó antes, este trabajo se enmarca en un proyecto iniciado recientemente que busca avanzar en el conocimiento de las ocupaciones humanas del noreste de Neuquén. Como punto de partida, se propuso un modelo arqueológico de base biogeográfica basado

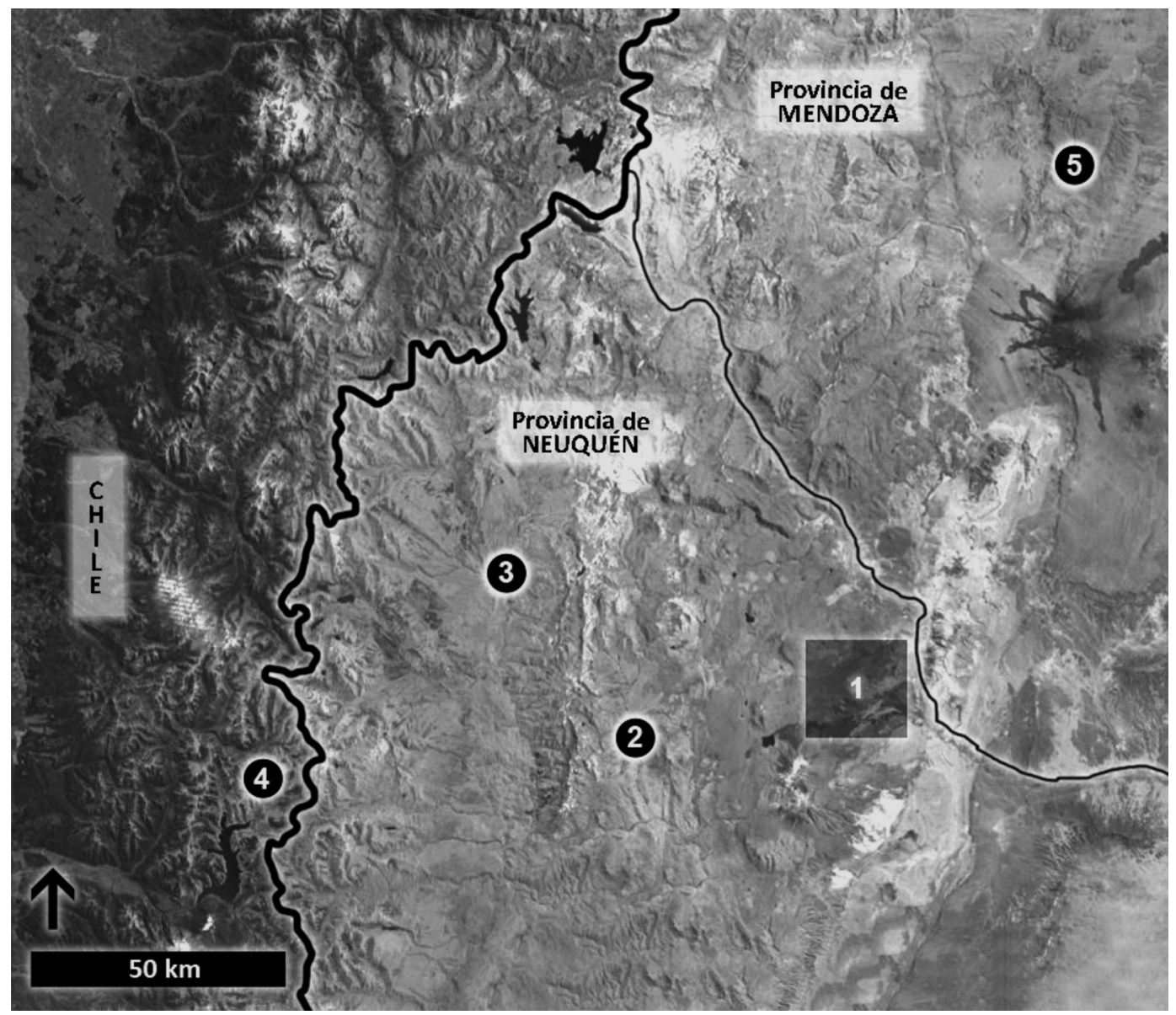

Figura 2. Áreas de investigación con representaciones rupestres consideradas en una escala macroregional. Referencias: 1 = Noreste de Neuquén, 2 = cuenca río Curi Leuvú (noroeste de Neuquén), 3 = cuenca río Neuquén (noroeste de Neuquén), 4 = Cordillera Andina de Linares (centro-este de Chile) y 5 = Departamento de Malargüe (sur de Mendoza). 
en información ecológica, climática y topográfica actual (Barberena 2013a). Específicamente, las variables biogeográficas consideradas fueron la capacidad de carga de herbívoros $(\mathrm{k})$, la topografía y la estacionalidad. A partir de la integración de las mismas se estratificó el paisaje estudiado en dos unidades espaciales con características diferenciales en función de su potencial para el uso y la circulación de las poblaciones humanas (Figura 3). Así, se observó que la mayor superficie del área de estudio corresponde a espacios de bajo k los cuales se ubican entre los 700 a los $1700 \mathrm{msnm}$ aproximadamente y están disponibles en forma anual. En contraposición, los espacios de alto k son aquellos ubicados entre los 1700 a los $2500 \mathrm{msnm}$, se encuentran geográficamente circunscriptos y, en nuestra región de trabajo, sólo permiten ocupaciones durante el verano.

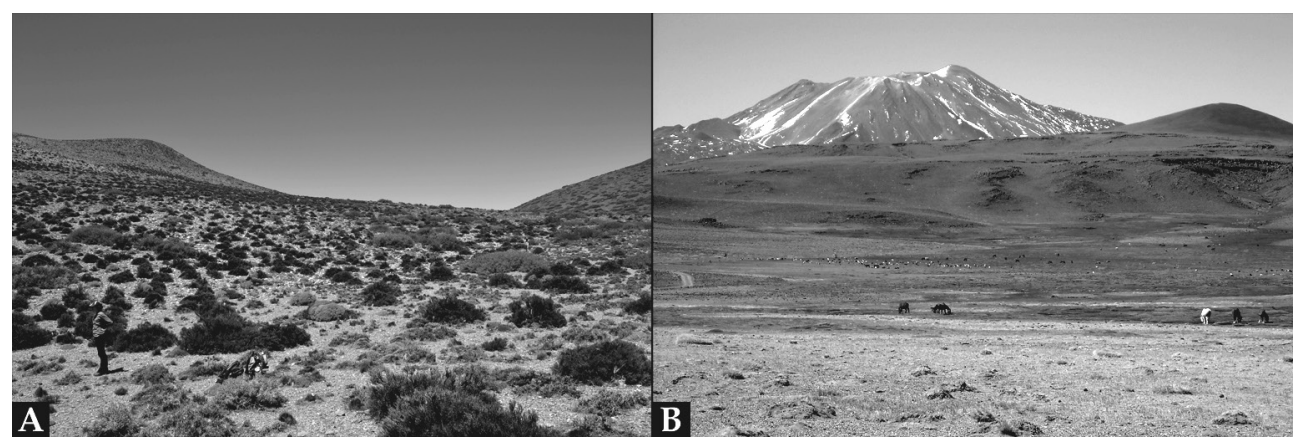

Figura 3. Espacios con diferentes propiedades biogeográficas de la región de estudio. A: Espacios de bajo k. B: Espacios de alto k.

Este análisis biogeográfico constituyó la base para la construcción de un modelo arqueológico a partir del cual se derivaron expectativas para los espacios mencionados en referencia a la intensidad ocupacional, la organización tecnológica, las formas de interacción social y la demarcación simbólica del espacio (Barberena 2013a). Éstas fueron planteadas, fundamentalmente, para una instancia de ocupación efectiva (sensu Borrero 1989-1990) en la historia de poblamiento de una región, en la cual se entiende que existe abundante información sobre la configuración del paisaje al tiempo que los rangos de acción o territorios ya se encuentran establecidos (Eerkens 1999). Asimismo, es esperable que en este contexto comiencen a operar mecanismos sociales dependientes de la densidad demográfica. Se entiende que esta instancia correspondería, a grandes rasgos, con el Holoceno tardío, aunque no necesariamente en forma exclusiva.

De acuerdo a lo anterior, se propuso que los espacios con alto $k$ de la región bajo estudio presentarían una ocupación más intensa y/o recurrente que aquellos con bajo k (Barberena 2013a). Así, en los primeros predominaría una estrategia tecnológica de aprovisionamiento del espacio y se favorecerían procesos de competencia, observándose en consecuencia, una intensa demarcación simbólica de los mismos en comparación con otros sectores. En contraposición, en los espacios de bajo k predominaría una estrategia tecnológica de aprovisionamiento de individuos y estos espacios no favorecerían condiciones de competencia, por lo cual, habrían sido demarcados simbólicamente con menor intensidad.

Asimismo, en una escala más amplia se propuso que la región bajo estudio en su conjunto, en función de su carácter marginal a nivel ecológico y geográfico, habría ocupado un rol de menor jerarquía en las redes de circulación y asentamiento humano de Patagonia septentrional en comparación con espacios vecinos (Barberena 2013a; Barberena et al. 2011). 
Hasta el momento se ha presentado una contrastación inicial del modelo a partir de la integración de diversas líneas de evidencia, entre las que se cuentan la paleoecología, la geoarqueología, la zooarqueología, la arqueobotánica, la tecnología lítica y cerámica y el arte rupestre (Barberena 2013a y b; Barberena et al. 2011, 2013; Belardi et al. 2013; Chidiak 2013; Llano y Barberena 2013; Pompei y Rughini 2013; Pompei et al. 2012; Romero y Re 2013). En muchos casos la información parece apoyar el modelo propuesto, mientras que en otros los datos disponibles para algunas líneas de evidencia complejizan el panorama (ver Discusión).

En el marco de estos trabajos se excavó Cueva Huenul 1, el sitio más importante en términos de representaciones rupestres (ver más adelante). En proximidad a estas últimas se excavaron cuatro cuadrículas (Barberena 2013b). En el sector más profundo del abrigo se emplazaron tres de ellas de $2 \times 1 \mathrm{~m}$, mientras que en una posición más cercana a la boca de la cueva se excavó la cuadrícula restante con una dimensión de 1 × $1 \mathrm{~m}$. Sobre la base de distintos fechados radiocarbónicos se pudo acotar la ocupación humana del sitio a dos lapsos cronológicos con un importante hiato entre ellos. Así, por un lado se cuenta el Componente 2 que corresponde al Holoceno temprano y abarca un lapso comprendido entre los 12.000 a 10.000 años cal. AP. Por otro lado, se encuentra el Componente 3 que corresponde al Holoceno tardío y consiste en un rango acotado entre los 1600 a 400 años cal. AP. Por su parte, el Componente 1 es paleontológico y corresponde al Pleistoceno final. A nivel sedimentario, este último se caracteriza por una elevada proporción de materia orgánica y fósforo disponible producto del aporte de excrementos de megafauna. Se infiere que las escasas evidencias arqueológicas recuperadas en el mismo se encuentran redepositadas por procesos de migración vertical desde los niveles superiores. Si bien preliminares, estas tendencias temporales proveen un marco cronológico inicial para contextualizar las ocupaciones humanas del área de estudio. Particularmente, dentro de los hallazgos correspondientes al Componente 3 (Holoceno tardío), interesa destacar que se registró la presencia de un fragmento de cáscara de calabaza ${ }^{1}$ pirograbado con motivos geométricos, caracoles marinos con manchas de un colorante rojo -posiblemente ocre-, así como un aumento en la frecuencia de pigmentos hallados en estratigrafía con respecto al Componente 2 (Llano y Barberena 2013; Barberena com. pers.).

Sobre la base de los antecedentes presentados y del modelo que enmarca este trabajo, se propone que durante el Holoceno tardío, dado el contexto de mayor interacción social y competencia por el espacio, sería esperable una alta intensidad en la circulación de información y, en consecuencia, una alta producción de arte rupestre en la región de estudio. Sin embargo, también se considera que sería esperable que la misma circule en forma diferencial entre espacios con características biogeográficas disímiles. En particular, sería esperable que los espacios de alto $\mathrm{k}$ hayan sido demarcados simbólicamente mediante una mayor intensidad en la ejecución de arte rupestre, a diferencia de lo observado en espacios con bajo $\mathrm{k}$.

Asimismo, en este trabajo también se comienza a evaluar la dinámica de circulación de información en una escala más amplia ya que, en función del rol marginal propuesto para el noreste de Neuquén dentro de las redes de circulación y asentamiento humano de Patagonia septentrional, es posible plantear una circulación de información de baja intensidad y más acotada en la región de estudio en comparación con lo observado para regiones con arte rupestre circundantes y, por lo tanto, características diferenciales de los registros rupestres. 


\section{Las representaciones rupestres}

\section{Distribución y densidad}

En las dos campañas realizadas hasta el momento $(2011,2012)$ se registraron cinco sitios con representaciones rupestres, todas ellas pintadas (Figuras 3 y 4). Dos de ellos, Paso de las Tropas 3 (PT3) y Cueva Yagui (CY), se ubican en diferentes sectores del cañadón del arroyo Buta Có. El primero se localiza en el curso superior a una altitud de $1800 \mathrm{msnm}$, mientras que el segundo se emplaza en el curso medio, a unos $1370 \mathrm{msnm}$. En ambos sitios, los motivos fueron ejecutados sobre paredones y bloques basálticos de textura lisa que, en el caso de CY, se distribuyen a lo largo de más de 20 m en asociación a una cueva de $10 \mathrm{~m}$ de largo aproximadamente. Cabe mencionar que tenemos noticias respecto de la ubicación de al menos otro sitio todavía no relevado en cercanía a PT3 que consiste en una pared con pinturas de color rojo que fue tempranamente documentada por Fernández (1974-1976: 16) para la localidad Paso de las Tropas².

A una altitud menor, cercana a los $1000 \mathrm{msnm}$, se ubica Cueva Huenul $1(\mathrm{CH} 1)$, cuyas grandes dimensiones $-18 \mathrm{~m}$ por $35 \mathrm{~m}$ - le confieren características de reparo altamente distintivas en la región (Figuras 4 y 5). La cueva fue originada por procesos erosivos, principalmente de carácter

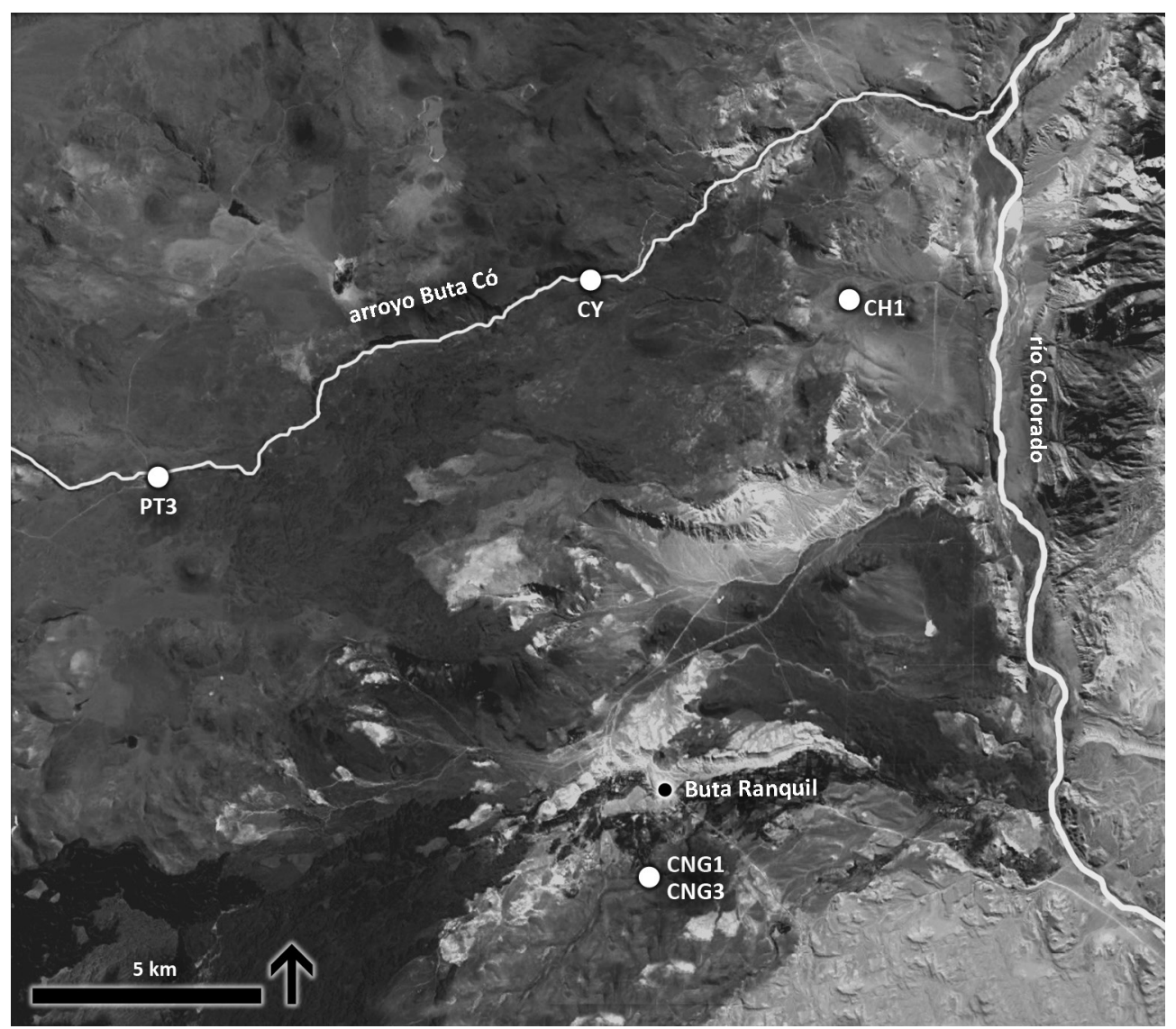

Figura 4. Ubicación de los sitios con representaciones rupestres analizados. 


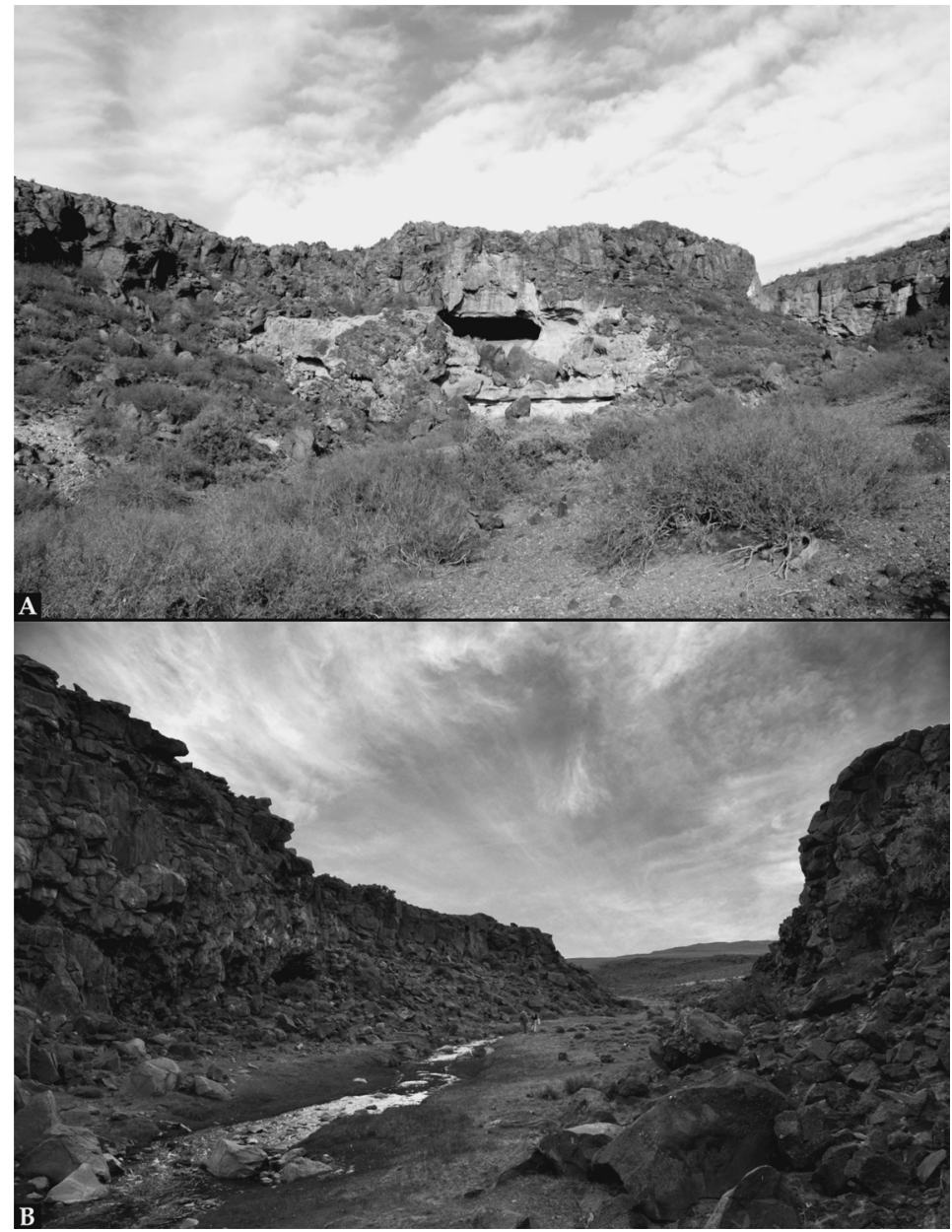

Figura 5. Ejemplos de tipos de sitios y soportes registrados. A: Cueva Huenul 1. B: Paso de las Tropas 3.

hídrico, que actuaron en el punto de contacto entre dos litologías de diferente tenacidad: una colada basáltica -Formación El Puente- que conforma el techo de la cueva e ignimbritas de textura rugosa -Formación Tilhué- que constituyen la base y paredes de la cueva (Narciso et al. 2004). Las pinturas se ejecutaron sobre éstas últimas pudiéndose identificar 11 paneles.

Por último, en el sur del área muestreada se relevaron otros dos sitios con pinturas rupestres, El Ciénego 1 y 3 (CNG1 y CNG3), en una altitud cercana a los $1180 \mathrm{msnm}$ (Figura 4). Se trata de dos cuevas pequeñas -de hasta $5 \mathrm{~m}$ de diámetro- en una barda basáltica de baja altura que, dada su proximidad, conforman una misma localidad arqueológica. Las filtraciones de agua que atraviesan ambas oquedades han afectado notablemente la conservación de las pinturas ubicadas en las paredes de estos sitios en particular.

En síntesis, la mayoría de los sitios relevados se localizan en un rango altitudinal que varía entre los 1000 a los $1400 \mathrm{msnm}$, a excepción de uno de ellos (PT3) que se ubica a una altitud mayor (1800 msnm), localizándose por tanto en un espacio de alto k. Llama 
la atención que los sitios analizados presentan características muy diversas en cuanto a sus condiciones de reparo y al tipo de soporte y roca disponible que, además, conllevan diferencias en términos de la exposición y visibilidad de las representaciones. Así, por ejemplo, las manifestaciones rupestres presentes en los sitios en cuevas -tales como CH1se encuentran reparadas y no son visibles desde el exterior de los abrigos, mientras que aquellas ubicadas en paredones y bloques al aire libre -como en PT3- están más expuestas $\mathrm{y}$, en consecuencia, son más visibles.

En los sitios mencionados se documentó un número mínimo de 243 motivos pintados que se distribuyen en forma heterogénea al interior del área de estudio (Tabla 1). Así, uno de ellos sólo cuenta con 4 motivos -PT3- ${ }^{3}$, mientras que otros como CNG1, CNG3 y CY registran frecuencias levemente mayores, de entre 20 y 55 motivos. Se destaca el sitio CH1 que con 140 motivos concentra el 57,61\% del total de la muestra analizada hasta el momento.

\begin{tabular}{|l|c|c|c|c|c|c|c|c|c|c|}
\cline { 2 - 11 } \multicolumn{1}{c|}{} & \multicolumn{2}{c|}{ Abstractos } & \multicolumn{2}{c|}{ Zoomorfos } & \multicolumn{2}{c|}{ Antrobomorfos } & \multicolumn{2}{c|}{ Indet. } & \multicolumn{2}{c|}{ Total } \\
\hline Sitios & $\mathrm{N}$ & $\%$ & $\mathrm{~N}$ & $\%$ & $\mathrm{~N}$ & $\%$ & $\mathrm{~N}$ & $\%$ & $\mathrm{~N}$ & $\%$ \\
\hline CH1 & 88 & $62.86 \%$ & 5 & $3.57 \%$ & 8 & $5.71 \%$ & 39 & $27.86 \%$ & 140 & $57.61 \%$ \\
\hline CNG1 & 16 & $80 \%$ & - & - & - & - & 4 & $20 \%$ & 20 & $8.23 \%$ \\
\hline CNG3 & 19 & $79.17 \%$ & 1 & $4.17 \%$ & - & - & 4 & $16.67 \%$ & 24 & $9.88 \%$ \\
\hline CY & 33 & $60 \%$ & 1 & $1.82 \%$ & - & - & 21 & $38.18 \%$ & 55 & $22.63 \%$ \\
\hline PT3 & 3 & $75 \%$ & - & - & - & - & 1 & $25 \%$ & 4 & $1.65 \%$ \\
\hline Total & 159 & $65.43 \%$ & 7 & $2.88 \%$ & 8 & $3.29 \%$ & 69 & $28.40 \%$ & 243 & $100 \%$ \\
\hline
\end{tabular}

Tabla 1. Cantidad de motivos y categorías por sitios del área de estudio ordenados altitudinalmente. Referencias: Indet. = Indeterminados.

\section{Categorías y tipos de motivos}

Entre las representaciones rupestres del área de estudio se observó una gran diversidad morfológica que fue organizada a partir de categorías generales y tipos de motivos. Dentro de las primeras, inicialmente se realizó una primera distinción entre motivos abstractos y figurativos. Así, en el área de estudio se observa que predominan ampliamente los motivos abstractos con el 65,43\% (N: 159), seguidos en muy baja frecuencia por los figurativos, que incluyen a los motivos zoomorfos $(2,88 \%, \mathrm{~N}$ : 7 ) y antropomorfos $(3,29 \%, \mathrm{~N}: 8)$ (Tabla 1 y Figura 6). Al considerarse separadamente los sitios identificados, se observa que en todos ellos predominan los motivos abstractos en frecuencias superiores al $60 \%$, alcanzando en algunos casos el $80 \%$ (Tabla 1). Dentro de los figurativos, los zoomorfos están presentes en 3 sitios (CH1, CY y CNG3) en frecuencias cercanas o menores al 4\%. Por su parte, llama la atención la distribución que presentan los antropomorfos ya que sólo se registran en el sitio $\mathrm{CH} 1(5,71 \%, \mathrm{~N}: 8)$. Por último, en el área de estudio se destaca un alto porcentaje de motivos indeterminados $(28,40 \%, \mathrm{~N}: 69)$ en función de los diversos procesos de deterioro natural que los afectan (insolación, filtraciones, acreciones, entre otros) 4 .

Posteriormente, esta variabilidad fue ordenada a partir de tipos de motivos definidos en función de rasgos morfológicos semejantes (Aschero 1988). Por un lado, dentro de los abstractos se relevaron formas geométricas como círculos (simples, concéntricos, con apéndices, etc.), líneas (rectas, quebradas, curvas y sinuosas) y puntos, todos ellos se presentan tanto en forma aislada como agrupados y/o alineados (Figuras 6 y 7). También 
se registraron figuras almenadas y escalonadas, gran variedad de líneas paralelas (rectas, sinuosas, zigzag, almenadas), diversos tipos de encadenados (rombos, triángulos y círculos) y figuras de simetría axial. Otros motivos abstractos se destacan por presentar una mayor complejidad y/o gran tamaño como algunas figuras escalonadas complejas y los cruciformes, entre los cuales hay algunos motivos que miden, por ejemplo, $1 \mathrm{~m}$ de alto y $0,60 \mathrm{~m}$ de ancho.

Por otro lado y a pesar de presentarse en muy baja frecuencia (N: 7), se destaca la variabilidad observada entre los zoomorfos ya que se definieron cuatro tipos de motivos (Figuras 6 y 8). Entre las siluetas se observan casos de ñandú y un zoomorfo indeterminado -posiblemente un camélido- y entre las pisadas se registran huellas de felino y tridígitos.

Por su parte, los antropomorfos también son muy escasos (N: 8) y el único tipo documentado es la figura humana (Figuras 6 y 8). En todos los casos, las figuras humanas aparecen vistas de frente pero mientras que en algunas se representaron los brazos rectos, en otras éstos fueron ejecutados semi-flexionados.
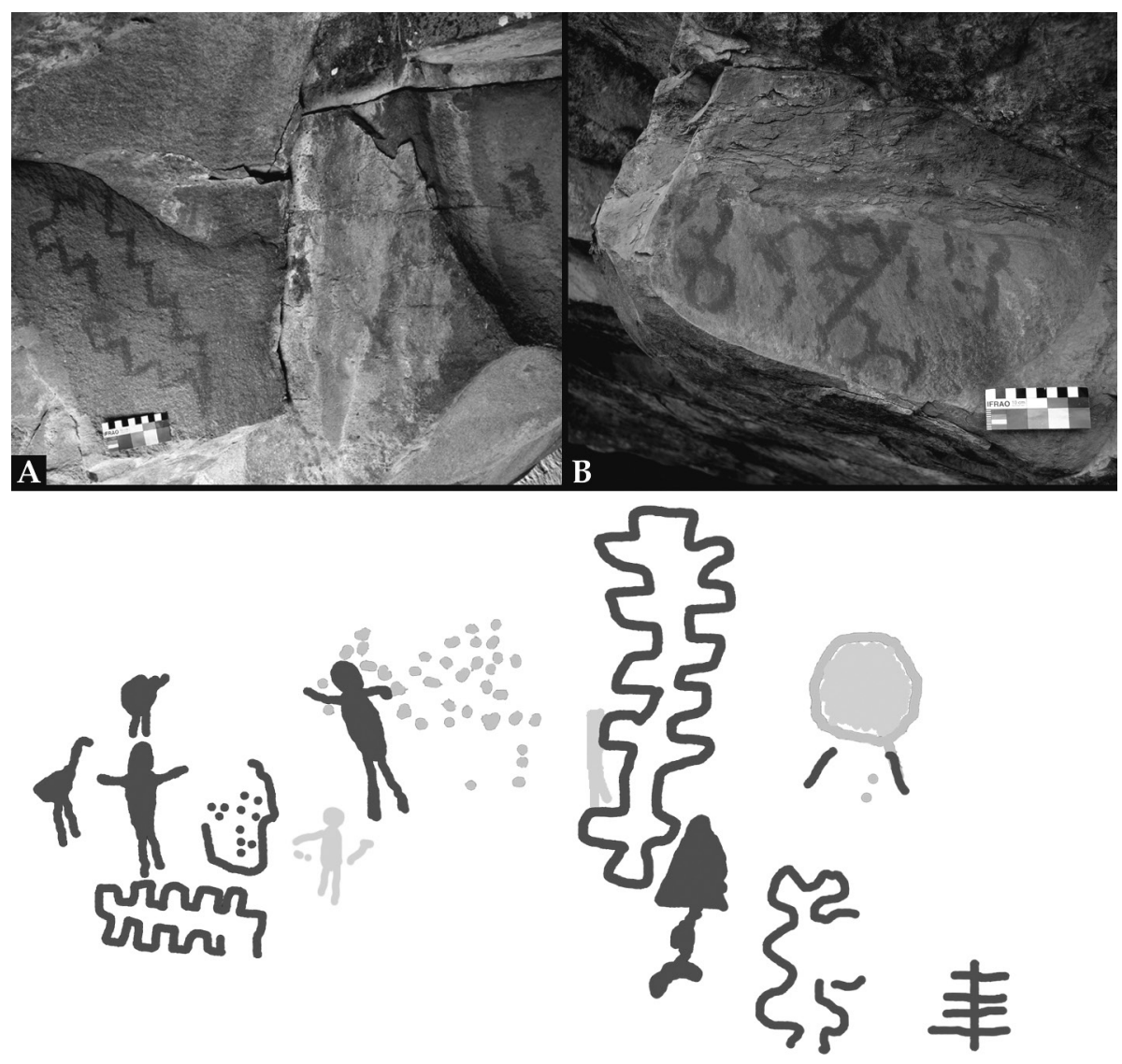

Blanco 10YR 8/1

Amarillo 2.5Y 8/6

Rojo 10R 5/8

aㅗㄴ.1

Figura 6. Ejemplos de representaciones rupestres documentadas en el noreste de Neuquén. Arriba: Cueva Yagui, A: UT 8 y B: UT 11. Abajo: CH1, Panel 8. 


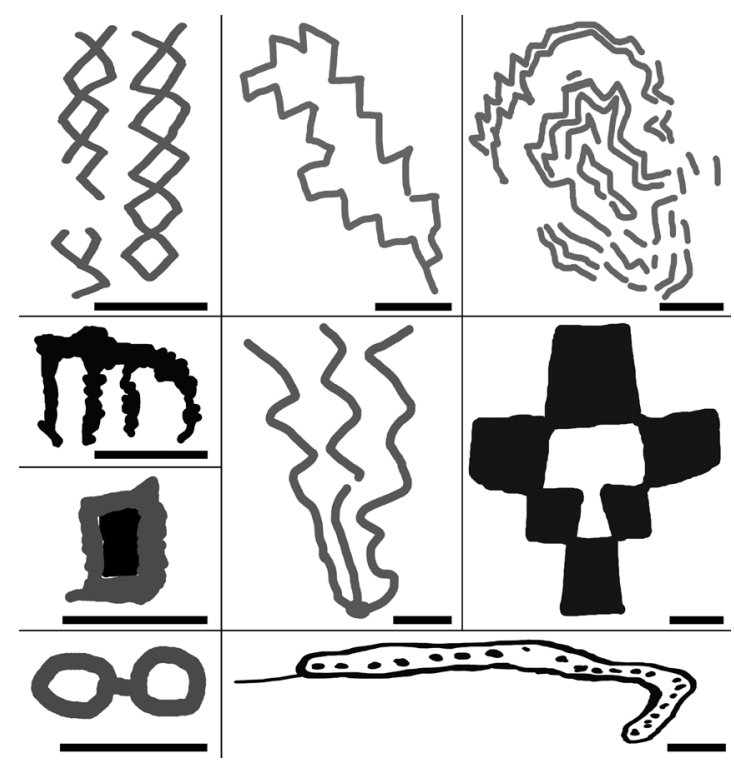

Figura 7. Ejemplos de tipos de motivos abstractos. Escala: $10 \mathrm{~cm}$.

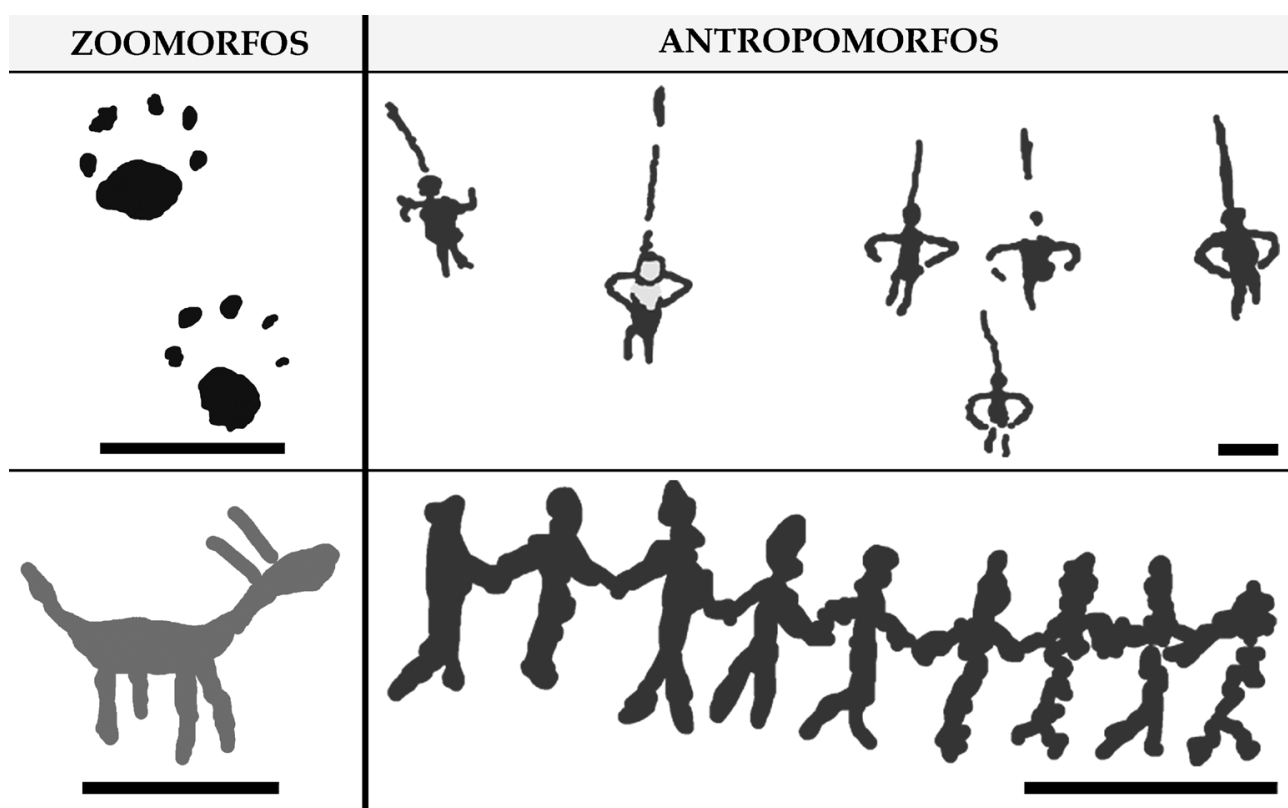

Figura 8. Ejemplos de tipos de motivos zoomorfos y antropomorfos. Escala: $10 \mathrm{~cm}$.

\section{Técnicas y tonalidades}

Como se adelantó, la totalidad de las representaciones rupestres relevadas corresponde a motivos pintados. El color más utilizado es el rojo dentro del cual se registraron hasta 4 tonos diferentes según la Munsell Soil Color Chart: rojo amarronado (10R 4/6) y rojo (10R 4/8, 10R 5/8 y 10R 6/8), entre los cuales predomina el tono 10R 5/8 (Figuras 6, 7 y 8 ) (Tabla 
2). En forma complementaria, también se registran motivos pintados en amarillo (2.5Y 8/6), blanco (10YR 8/1 y 8/2) y negro (10R 4/1). Se destaca CH1 que concentra toda la variabilidad de colores registrados para el área. Asimismo, si bien se observan diferentes grados de desvaído entre las pinturas estudiadas, aún se necesita profundizar en las investigaciones para determinar si responden a cuestiones de deterioro o a una cronología diferencial.

\begin{tabular}{|l|c|c|c|c|}
\cline { 2 - 5 } \multicolumn{1}{c|}{} & \multicolumn{4}{c|}{ Colores } \\
\hline Sitios & Blanco & Amarillo & Roio & Negro \\
\hline CH1 & $\mathrm{x}$ & $\mathrm{x}$ & $\mathrm{x}$ & $\mathrm{x}$ \\
\hline CNG1 & - & $\mathrm{x}$ & $\mathrm{x}$ & - \\
\hline CNG3 & - & - & $\mathrm{x}$ & - \\
\hline CY & - & - & $\mathrm{x}$ & $\mathrm{x}$ \\
\hline PT3 & - & - & $\mathrm{x}$ & - \\
\hline
\end{tabular}

Tabla 2. Presencia (X) / Ausencia (-) ae colores registraaos por sitios.

Por otra parte, la mayoría de los motivos son monocromos (Figuras 6, 7 y 8). Excepcionalmente, en los sitios CH1, CY y CNG1 se registraron algunos casos de bicromía (N: 10, 4,11\%) donde se combinan, por ejemplo, los colores rojo-negro, amarillo-blanco y rojo-amarillo.

También se registró el predominio de motivos con tratamiento lineal aunque se relevaron ejemplos ejecutados mediante tratamiento plano (N: 32, 13,17\%). Este es el caso de varios motivos figurativos, como el ñandú y las pisadas de felino, y en menor medida, de algunos motivos abstractos como los círculos y cruciformes.

\section{Superposiciones}

Se registró un total de 26 casos de superposiciones en tres de los cinco sitios considerados: CNG1, CNG3 y CH1. No obstante, casi todas ellas se concentran en este último (N: 23). La mayor parte de las superposiciones ( $\mathrm{N}$ : 20, 76,92\%) involucran motivos abstractos de diferentes colores sobre otros de la misma categoría (Figura 6) (Tabla 3). Entre éstos se cuentan ejemplos de círculos y puntos agrupados y alineados así como también rombos encadenados, líneas paralelas, figuras almenadas y cruciformes. Resalta la presencia de motivos figurativos formando parte de las superposiciones, todos ellos ejemplos de figuras humanas que ocupan siempre la posición superior (N: 6, 23,08\%).

\begin{tabular}{|c|l|c|c|c|c|c|c|}
\cline { 3 - 8 } \multicolumn{2}{c|}{} & \multicolumn{6}{|c|}{ Motivos inferiores } \\
\cline { 3 - 9 } \multicolumn{1}{c|}{} & ABS & ZOO & ANT & INDET & Total \% & Total N \\
\hline \multirow{4}{*}{$\begin{array}{c}\text { Motivos } \\
\text { superiores }\end{array}$} & $76.92 \%$ & - & - & - & $76.92 \%$ & 20 \\
\cline { 2 - 8 } & ZOO & - & - & - & - & - & - \\
\cline { 2 - 8 } & ANT & $23.08 \%$ & - & - & - & $23.08 \%$ & 6 \\
\cline { 2 - 8 } & INDET & - & - & - & - & - & - \\
\cline { 2 - 8 } & Total \% & $100 \%$ & - & - & - & $100 \%$ & 26 \\
\cline { 2 - 8 } & Total N & 26 & - & - & - & 26 & \multicolumn{1}{c}{}
\end{tabular}

Tabla 3. Categorías de motivos involucrados en superposiciones. Referencias: $A B S=$ Abstractos, $\mathrm{ZOO}=$ Zoomorfos, ANT $=$ Antropomorfos e Indet $=$ Indeterminados 


\section{Discusión y consideraciones finales}

Las representaciones rupestres del noreste de Neuquén analizadas corresponden en su totalidad a pinturas, entre las que predominan los motivos abstractos monocromos de color rojo y de tratamiento lineal. No obstante ello, llama la atención la presencia -aunque en muy baja proporción- de motivos zoomorfos en tres de los cinco sitios estudiados así como de antropomorfos en uno de ellos, además de los contados casos de motivos bícromos o ejecutados en color blanco, amarillo o negro.

Preliminarmente, se sugiere que la mayor parte de las pinturas del área de estudio pueden ser asignadas al Holoceno tardío y, más específicamente, a los últimos 1600 años. Esta sugerencia se apoya, por un lado, en el marco cronológico provisto por los hallazgos previamente comentados en el Componente 3 de CH1 (Barberena 2013b). Por otro lado, también se toma en consideración que algunos de los tipos de motivos registrados en el área de estudio, como las paralelas, los encadenados y las figuras de simetría axial, son similares a otros registrados en áreas cercanas y que fueron asignados por otros investigadores a momentos tardíos -entre los siglos VIII y XV de nuestra era- (ver citas en Gradin 19971998). Sin embargo, no se descarta la existencia de diversos momentos de ejecución, dado las superposiciones, la cantidad de representaciones documentadas en algunos sitios y la variedad de motivos registrados.

En este trabajo se busca iniciar la discusión respecto del potencial rol del arte rupestre analizado como demarcador simbólico del espacio en relación con el modelo biogeográfico propuesto para el área de estudio en una escala mesoregional (Barberena 2013a). Así, al retomar los espacios con diferentes propiedades biogeográficas (alto y bajo k), se observa que aquellos con bajo k-amplios y disponibles anualmente-, concentran la mayor densidad de representaciones rupestres y sitios identificados hasta el momento (239 motivos en cuatro sitios). Asimismo, estos últimos reúnen toda la variabilidad de categorías de motivos y tonalidades relevadas, así como también todos los casos de superposiciones (N: 26). Por lo tanto, en esta primera instancia esta línea de evidencia sugiere una circulación de información diferencial en esta escala y, además, que ésta habría sido más intensa en los espacios de bajo $\mathrm{k}$ de uso anual.

De esta manera, la información generada a partir de las representaciones rupestres da cuenta de una jerarquización diferencial del espacio, en términos de la circulación de información, para las poblaciones humanas que habitaron el noreste de Neuquén en el pasado.

En principio, los resultados observados se contraponen con las expectativas derivadas del modelo marco de esta investigación, que sugiere que durante el Holoceno tardío, los espacios de alto $\mathrm{k}$-espacial y temporalmente circunscriptos-, habrían favorecido procesos de competencia y, consecuentemente, habrían sido demarcados simbólicamente con una mayor intensidad en comparación con otros sectores del área de estudio. Este panorama nos dispara varios interrogantes que serán explorados en un futuro cercano: ¿existe un sesgo en el muestreo? ¿qué indican las otras líneas de evidencia arqueológicas asociadas a los motivos rupestres? ¿existieron otros medios que pudieron haber expresado los procesos de competencia inferidos a partir del modelo para una escala mesoregional? ¿las inferencias del modelo sólo son aplicables en una escala espacial amplia (macroregional)? Se espera que los nuevos trabajos de campo así como la profundización en el análisis de las representaciones 
rupestres del área permitan responder éstos y otros nuevos interrogantes generados a partir de las tendencias preliminares presentadas. Aunque el modelo genera expectativas para distintas escalas espaciales, resulta probable que su contrastación requiera una escala espacial de análisis más amplia, que incluya espacios de alto k que sean tanto estacionales -como los aquí tratados- como de uso anual -tal como las vecinas cuencas de los ríos Neuquén y Curi Leuvú- (cf. Fernández 2000 [1979]; Hajduk y Cúneo 2009). Por otra parte, cabe señalar que el modelo es de grano grueso y no contempla que hay sectores dentro de los espacios de bajo $\mathrm{k}$ que pueden haber funcionado como espacios de alto k, dada la disponibilidad diferencial de agua. Este puede ser el caso de la cuenca del arroyo Buta Có.

\begin{tabular}{|c|c|c|c|l|c|}
\cline { 2 - 6 } \multicolumn{1}{c|}{} & $\begin{array}{c}\text { Altitud } \\
\text { (msnm) }\end{array}$ & $\begin{array}{c}\mathrm{N} \\
\text { sitios }\end{array}$ & $\begin{array}{c}\mathrm{N} \\
\text { motivos }\end{array}$ & Técnicas & $\begin{array}{c}\text { Categorías } \\
\text { de motivos }\end{array}$ \\
\hline $\begin{array}{c}\text { Noreste de } \\
\text { Neuquén }\end{array}$ & $\begin{array}{c}1000 \\
\text { a } 1800\end{array}$ & 5 & 243 & Pintura & $\begin{array}{c}\text { ABS }(65,43 \%) \\
\text { ANT }(3,29 \%) \\
\text { ZOO }(2.88 \%)\end{array}$ \\
\hline $\begin{array}{c}\text { Cuenca río } \\
\text { Curi Leuvú } \\
\text { (NO Neuquén) }\end{array}$ & SD & 11 & SD & $\begin{array}{c}\text { Pintura } \\
\text { Grabado }\end{array}$ & $\begin{array}{c}\text { predominio ABS } \\
\text { escasos ANT, ZOO }\end{array}$ \\
\hline $\begin{array}{c}\text { Cuenca río } \\
\text { Neuquén } \\
\text { (NO Neuquén) }\end{array}$ & $\begin{array}{c}\text { mayoría } \\
1700 \\
\text { a } 2000\end{array}$ & 12 & 329 & Grabado & $\begin{array}{c}\text { escasos ANT, ZOO } \\
\text { predominio ABS }\end{array}$ \\
\hline $\begin{array}{c}\text { Cordillera Andina } \\
\text { de Linares } \\
\text { (CE de Chile) }\end{array}$ & $\begin{array}{c}2100 \\
\text { a } 2600\end{array}$ & 16 & 3074 & Grabado & $\begin{array}{c}\text { ABS (54,07\%) } \\
\text { ANT (35\%) } \\
\text { ZOO (9.5\%) }\end{array}$ \\
\hline $\begin{array}{c}\text { Departamento } \\
\text { de Malargüe } \\
\text { (sur de Mendoza) }\end{array}$ & SD & 17 & SD & $\begin{array}{c}\text { Pintura } \\
\text { Grabado }\end{array}$ & $\begin{array}{c}\text { predominio ABS } \\
\text { escasos ANT, ZOO }\end{array}$ \\
\hline
\end{tabular}

Tabla 4. Áreas de investigación con representaciones rupestres consideradas en una escala macroregional. Referencias: $\mathrm{NO}=$ noroeste, $\mathrm{CE}=$ centro-este, $\mathrm{SD}=$ Sin datos, $\mathrm{ABS}=$ Abstractos, $\mathrm{ANT}=$ Antropomorfos y ZOO = Zoomorfos. Ver referencias bibliográficas de cada área en el texto.

También es interesante realizar una primera comparación de la información generada para el área de estudio en una escala macroregional, contrastándola con aquella publicada por otros investigadores para áreas con arte rupestre ubicadas a menos de $100 \mathrm{~km}$ lineales pero con características geográficas y ecológicas diferentes como la Cordillera Andina de Linares - centro-este de Chile -, las cuencas de los ríos Neuquén y Curi Leuvú - noroeste de Neuquén - y el sur de Mendoza - departamento Malargüe - (Fernández 2000 [1979]; Gradin 1997-1998; Hajduk y Cúneo 2009; Menghin 1957; Niemeyer y Weisner 1972-1973; Schobinger 1956, 1978, 2002; Tucker y Risi 2010) (Figura 2 y Tabla 4). Cabe aclarar que no se dispone de información cuantitativa para todas las áreas consideradas. Así, gran parte de esta comparación de carácter inicial debe hacerse en términos cualitativos. 
En primer lugar, en esta macroregión se evidencia un amplio repertorio de motivos grabados y pintados, entre los cuales predominan los abstractos por sobre los figurativos. Se planteó una cronología tardía para la mayoría de ellos -entre los siglos VIII y XV de nuestra era- (Gradin 1997-1998), lo cual coincide en términos generales con aquella propuesta para el área abordada en este trabajo.

Se observan tanto similitudes como diferencias entre las representaciones rupestres de las áreas comparadas. En primer lugar, en todas las áreas consideradas se observa el predominio de los motivos abstractos por sobre los figurativos, a pesar que la proporción es variable en cada una de ellas. También se destaca que todas las áreas comparten determinados tipos de motivos, entre los que se incluyen una diversidad de líneas paralelas, figuras de simetría axial, puntos, encadenados, cruciformes, almenados y escalonados. Algunos de ellos han sido asignados por otros autores a los estilos de paralelas y grecas (sensu Menghin 1957).Las similitudes observadas indican que el área de estudio formó parte de la dinámica de circulación de información, en escala macroregional, junto a otros espacios que difieren a nivel geográfico y ecológico. Distintas líneas de evidencia remarcan esta observación, principalmente la distribución de ciertos tipos de cerámica y de tipos químicos de obsidiana con procedencia del Maule (Cortegoso et al. 2013).

No obstante, observamos que el área de estudio se diferencia de las otras regiones consideradas por presentar una menor cantidad de sitios y motivos, si bien se debe tener presente el inicio reciente de las investigaciones en el noreste de Neuquén y el carácter preliminar de la muestra analizada. También notamos que, al igual que en nuestra área de trabajo, las representaciones rupestres registradas en la cuenca del río Curi Leuvú se localizan principalmente en áreas de baja altitud y uso anual -invernada-, a diferencia de lo observado para la cuenca del río Neuquén y la Cordillera Linares donde las manifestaciones rupestres se emplazan en ámbitos de uso exclusivamente estival -veranada-. Asimismo, en nuestra región de estudio se registró una única técnica de ejecución -pintura-, mientras que en las áreas vecinas se registran otras -grabado- o ambas -pintura y grabado-. Otras particularidades del área de estudio remiten al uso de varios colores y a la presencia de diversos diseños de figuras humanas, que deberán ser evaluadas a medida que avancen las investigaciones.

De esta manera, las diferencias registradas en términos de la densidad de sitios y motivos permiten sostener la hipótesis de un rol marginal del noreste de Neuquén en la dinámica de circulación de información en una escala macroregional. A futuro, se podrá contribuir a identificar vectores geográficos de circulación de información, así como tendencias temporales (Belardi et al. 2013).

Las tendencias preliminares presentadas comienzan a llenar un vacío en la información de la que se disponía para el noreste neuquino. A futuro, esperamos realizar más trabajos de campo para documentar nuevos sitios con representaciones rupestres y revisar los ya relevados. Asimismo, también buscaremos profundizar en el análisis de la información aquí presentada, prestando especial atención a la definición de tipos de motivos a partir de los cuales se podrá ahondar en las comparaciones preliminarmente efectuadas, tanto en la escala meso como en la macroregional. Estos avances nos permitirán profundizar en las temáticas discutidas y complejizar el panorama descripto. Así, a partir de la correlación de esta línea de evidencia arqueológica con diversos indicadores de intensidad de uso y 
de las condiciones climático-ambientales específicas (cf. McDonald y Veth 2011), se espera generar información que permita modelar los cambios observados en los roles adoptados por el arte rupestre y, consecuentemente, en el tipo de información trasmitida por el mismo en referencia a la historia ocupacional de la región de estudio.

Agradecimientos: Esta publicación es producto del Proyecto PICT Bicentenario 2010-1856 (Agencia Nacional de Promoción de la Ciencia y la Tecnología, Argentina). Agradecemos especialmente a las autoridades de Buta Ranquil y Barrancas, provincia de Neuquén (Juvenal Urrutia, Hugo Zalazar, Gabriel Barros, Paulina Valenzuela, Marcela Carrera, Nora Vázquez y Roque Díaz), y a los arqueólogos de la Secretaría de Cultura de Neuquén (Claudia Della Negra, Estela M. Cúneo, Liliana Martínez y Pablo Azar). Un agradecimiento especial a Raúl Vázquez, Manu, Luis, Diego y Ramiro por colaborar con el relevamiento. A Ramiro por sus observaciones. Finalmente, agradecemos a los dos evaluadores anónimos cuyos comentarios y sugerencias contribuyeron a mejorar la calidad de este trabajo.

\section{Notas}

1 El resto corresponde a un fragmento de epicarpo del taxón doméstico Lagenaria sp. de la familia Cucurbitaceae (Llano y Barberena 2013).

2 "Paso de las Tropas, Departamento Chos Malal. Latitud y longuitud aproximadas, intersección del paralelo $37^{\circ}$ sur y meridiano $70^{\circ}$ oeste. Se halla entre los volcanes Huayle y Tromen, cabeceras del arroyo de Butacó, tributario del río Colorado. Altura sobre el mar: $1800 \mathrm{~m}$. Se trata de un afloramiento grande de rocas basálticas; en una leve concavidad del frente rocoso, con orientación al este, se encuentra una inscripción de aproximadamente 0,40 $\mathrm{m} 2$ de superficie (Figura 6). Los trazos, aparentemente aplicados mediante pincel, son bastante finos $(1 \mathrm{~cm})$. El color empleado ha sido el rojo. Dibujos similares han sido observados en el sur de Mendoza." (Fernández 1974-1976: 16) (el resaltado es nuestro). Se hace notar que en este pasaje el autor reporta la existencia de una única unidad topográfica para el sitio Paso de las Tropas en la cual identificó sólo un motivo rupestre.

3 Cabe aclarar que, por lo que se desprende del texto de Fernández (1974-1976) anteriormente citado así como de la única foto publicada por este autor, el número de motivos presentes en el sitio Paso de las Tropas podría ascender a un máximo de N: 6.

4 Se aclara que la cantidad de motivos indeterminados podría variar a futuro en función de la aplicación de programas digitales (como el Adobe Photoshop y el Image J-DStretch) que permiten una mejor visualización de los motivos desvaídos.

\section{Bibliografía citada}

Aschero, C.A.

1988 Pinturas rupestres, actividades y recursos naturales: un encuadre arqueológico. Arqueología Contemporánea Argentina. Actualidad y Perspectivas (ed. por H. Yacobaccio), pp. 109-145. Ediciones Búsqueda, Buenos Aires.

Aunger, R.

2009 Human Communication as Niche Construction. Pattern and Process in Cultural Evolution (ed. por S. Shennan), pp. 33-43. University of California Press, Berkeley. 
Barberena, R.

2010. Arqueología del norte de Neuquén: geografía humana, tendencias temporales y procesos demográficos. Proyecto PICT 2010-1856 presentado ante el CONICET. Buenos Aires.

2013a Biogeografía, competencia y demarcación simbólica del espacio: modelo arqueológico para el norte de Neuquén. Intersecciones en Antropología 14: 5-19.

2013b Discordancias y Discontinuidades en Patagonia septentrional: crono-estratigrafía de Cueva Huenul 1 (Neuquén, Argentina). Arqueología en ambientes de altura en Mendoza y San Juan (ed. por V. Cortegoso y V. Durán). EDIUNC, Universidad Nacional de Cuyo Editorial UNCuyo, Mendoza. En prensa.

Barberena, R., A. Hajduk, A. Gil, G. Neme, V. Durán, M.D. Glascock, M. Giesso, K. Borrazzo, M.P. Pompei, M.L. Salgán, V. Cortegoso, G. Villarosa y A.A. Rughini

2011 Obsidian in the south-central Andes: geological, geochemical, and archaeological assessment of north Patagonian Sources (Argentina). Quaternary International 245: 25-36.

Barberena, R., M.P. Pompei, A.A. Rughini, M. Chidiak, G. Romero y L. Acuña

2013. El poblamiento humano del Norte de Neuquén: procesos y discontinuidades. Actas del XVIII Congreso Nacional de Arqueología Argentina (ed. por J.R. Bárcena y S.E. Martín), pp. 520. Universidad Nacional de La Rioja, La Rioja.

Barton, C.M., G.A. Clark y A.E. Cohen

1994. Art as information: explaining Upper Paleolithic art in Western Europe. World Archaeology 26 (2): 185-207.

Belardi, J.B., R. Barberena, R. Goñi y A. Re

2013. The Development of a Legacy: Evolution, Biogeography and Archaeological Landscapes. Darwin's Legacy: The Status of Evolutionary Archaeology in Argentina. Tribute to the 200th anniversary of the birth of Charles Darwin and the 150th anniversary of the publication of The Origin of Species (ed. por M. Cardillo y H. Muscio), pp. 89-98. IMHICIHU-CONICET, Buenos Aires.

Borrero, L.A.

1989-1990. Evolución cultural divergente en la Patagonia austral. Anales del Instituto de la Patagonia (Serie Ciencias Sociales) 19: 133-139.

Bradley, R., F. Criado Boado y R. Fabregas Vaicarce

1994. Rock art research as landscape archaeology: a pilot study in Galicia, north-west Spain. World Archaeology 25 (3): 374-390.

Chidiak, M.

2013 Tecnología cerámica en el Noreste de Neuquén (Dpto. Pehuenches): un enfoque preliminar. Actas del XVIII Congreso Nacional de Arqueología Argentina (ed. por J.R. Bárcena y S.E. Martín), pp. 522. Universidad Nacional de La Rioja, La Rioja.

Cortegoso, C., M.D. Glascock, A.M. De Francesco, V. Durán, G. Neme, A. Gil, M. Giesso, L. Sanhueza, L. Cornejo, R. Barberena y M. Bocci

2013. Chemical Characterization of Obsidian in Central Western Argentina and Central Chile: Archaeological Problems and Perspectives. Physical, Chemical and Biological Markers in Argentine Archaeology: Theory, Methods and Applications (ed. por D. Kligmann y M. Morales). British Archaeological Reports, Archaeopress, Oxford. En prensa. 
David, B. y H. Lourandos

1998 Rock art and socio-demography in northeastern Australian prehistory. World Archaeology 30 (2): 193-219.

Dincauze, D.

2000. Environmental Archaeology, Principles and Practice. Cambridge University Press, Cambridge.

Durán, V., M. Giesso, M.D, Glascock, G. Neme, A. Gil y L. Sanhueza

2004 Estudio de fuentes de aprovisionamiento y redes de distribución de obsidiana durante el Holoceno Tardío en el sur de Mendoza (Argentina). Estudios Atacameños 28: 25-43.

Eerkens, I. W.

1999 Common-pool resources, buffer zones, and jointly-owned territories: Hunter gatherer land and resource tenure in Fort Irwin, Southeastern California. Human Ecology 27: 188-213.

Fernández, J.

1974-1976 Estudios sobre el arte rupestre de la provincia del Neuquén. Anales de Arqueología y Etnología, Volumen 29-31: 5-36.

1978 Corpus de arte rupestre neuquino. $1^{\circ}$ parte. Revista del Museo Provincial 1: 17-93.רר

2000 [1979] Las piedras con marcas de la cordillera del Viento. Arte rupestre en el departamento Minas, Neuquén, Argentina. Sociedad Argentina de Antropología, Buenos Aires.

Folguera, A., G. Bottesi, T. Zapata y V.A. Ramos

2008 Crustal collapse in the Andean back arc since 2 Ma: Tromen volcanic plateau, Southern Central Andes ( $36^{\circ} 40^{\prime} 0-37^{\circ} 30^{\prime}$ S). Tectonophysics 459: 140-160.

Galland, O., E. Hallot, P.R. Cobbold, G. Ruffet y J. de Bremond d'Ars

2007. Volcanismin a compressional Andean setting: a structural and geochronological study of Tromen volcano (Neuquen province, Argentina). Tectonics 26: 1-24.

Gradin, C.J.

1997-1998. El arte rupestre del sur mendocino entre los siglos VIII y XV de la era ¿Un área de conflicto o de convivencia? Relaciones de la Sociedad Argentina de Antropología XXII-XXIII: 7-23.

Hajduk, A. y E.M. Cúneo

2009. Representaciones rupestres en la Cuenca del río Curi Leuvú (departamento Chos Malal, Provincia del Neuquén, República Argentina). Informe preliminar. Arqueología de la Patagonia. Una mirada desde el último confín (ed. por M. Salemme, F. Santiago, M. Álvarez, E. Piana, M. Vázquez y E. Mansur), pp. 515-526. Editorial Utopías, Ushuaia.

Jochim, M.

1983. Paleolithic cave art in ecological perspective. Hunther-gatherer economy in prehistory (ed. por G. Bailey), pp. 212-219. Cambridge University Press, Cambridge.

Llano, C. y R. Barberena

2013. Explotación humana de especies vegetales en Patagonia Septentrional: el registro arquebotánico de Cueva Huenul 1 (Provincia de Neuquén, Argentina). Darwiniana nueva serie 1 (1): 5-19. 
McDonald, J. y P. Veth

2011 Information exchange among Hunter-Gatherers of the western desert of Australia. Information and its Role in Hunter-Gatherer Bands (ed. por R. Whallon, W.A. Lovis y R.K. Hitchcock), pp. 221-233. Cotsen Institute of Archaeology Press, USA.

2013 Rock art in arid landscapes: Pilbara and Western Desert petroglyphs Australian Archaeology 77: 1-16.

Menghin, $\mathrm{O}$.

1957 Estilos del Arte Rupestre de Patagonia. Acta Praehistorica I.

Morrone, J.J.

2001 Biogeografía de América Latina y el Caribe. M\&T-Manuales \& Tesis SEA, Volumen 3, Zaragoza.

Narciso, V., G. Santamaría y J. C.M. Zanettini

2004 Hoja Geológica 3769-I, Barrancas. Provincias de Mendoza y Neuquén. Boletín del Instituto de Geología y Recursos Minerales 253. SEGEMAR, Buenos Aires.

Niemeyer, H. y L. Weisner

1972-1973 Los petroglifos de la cordillera andina de Linares (Provincias de Talca y Linares, Chile). Actas del VI Congreso de Arqueología Chilena Volumen II, pp. 405-470. Universidad de Chile, Santiago de Chile.

Páez, M., F. Quintana y C. Pérez

2004 Biogeografía de las regiones áridas y semiáridas entre $35^{\circ}$ y $39^{\circ} \mathrm{S}$, Argentina. Boletín de la Sociedad Argentina de Botánica 39 (3-4): 171-180.

Pompei, M.P. y A. Rughini

2013 Análisis de la variabilidad de la tecnología lítica del noreste de Neuquén (Localidad Buta Ranquil-Barrancas). Actas del XVIII Congreso Nacional de Arqueología Argentina (ed. por J.R. Bárcena y S.E. Martín), pp. 521. Universidad Nacional de La Rioja, La Rioja.

Pompei, M.P., R. Barberena, M.E. de Porras, K. Borrazzo, A. Rughini y A. Gil

2012 Late Quaternary Ecosystems and Humans in Northern Patagonia (Neuquén, Argentina). Current Research in the Pleistocene, Volumen especial: Southbound, Late Pleistocene Peopling of Latin America (ed. por L. Miotti, M. Salemme, N. Flegenheimer y T. Goebel), pp. 187-190. Center for the Study of the First Americans, Texas A\&M University, Texas.

Re, A.

2010 Representaciones rupestres en mesetas altas de la Provincia de Santa Cruz. Circulación de información en espacios de uso estacional. Tesis Doctoral. Universidad de Buenos Aires, Buenos Aires.

Romero, G. y A. Re

2013 Representaciones rupestres del noreste de Neuquén (Depto. Pehuenches). Actas del XVIII Congreso Nacional de Arqueología Argentina (ed. por J.R. Bárcena y S.E. Martín), pp. 522. Universidad Nacional de La Rioja, La Rioja. 
Schobinger, J.

1956 El arte rupestre de la Provincia de Neuquén. Anales de Arqueología y Etnología de la Universidad Nacional de Cuyo XII: 115-227.

1978 Nuevos sitios de arte rupestre en el Departamento Malargüe de Mendoza. Relaciones de la Sociedad Argentina de Antropología XII: 175-182.

1985 Áreas intermedias o marginales. Cazadores de la Patagonia y agricultores andinos. Arte rupestre de la Argentina (ed. por J. Schobinger y C.J. Gradin), pp. 80-91. Encuentro Ediciones, Madrid.

2002 Arte rupestre del departamento de Malargüe. Entre Montañas y Desiertos: Arqueología del Sur de Mendoza (ed. por A. Gil y G. Neme), pp. 103-118. Sociedad Argentina de Antropología, Buenos Aires.

Scheinsohn, V.

2011 Rock Art Information among Hunter-Gatherers in Northwest Patagonia: An Assesment of Environmental and Territorial Models. Information and its Role in Hunter-Gatherer Bands (ed. por R. Whallon, W.A. Lovis y R.K. Hitchcock), pp. 235-247. Cotsen Institute of Archaeology Press, USA.

Taçon, P.

1994 Socialising landscapes: the long-term implications of signs, symbols and marks on the land. Archaeology in Oceania 29: 117-129.

Tucker, H. y A. Risi

2010 El registro simbólico como codificación del paisaje. Aproximaciones al estudio del arte rupestre del departamento de Malargüe, Mendoza, Argentina. Libro de resúmenes VIII Simposio Internacional de Arte Rupestre, pp. 117-120. ISES-UNT, San Miguel de Tucumán. 